\title{
PEI HSU
}

\section{WILFRID S. KENDALL}

\section{Limiting angle of brownian motion in certain two- dimensional Cartan-Hadamard manifolds}

Annales de la faculté des sciences de Toulouse $\sigma^{e}$ série, tome $1, \mathrm{n}^{\circ} 2$ (1992), p. 169-186

<http://www.numdam.org/item?id=AFST_1992_6_1_2_169_0>

(C) Université Paul Sabatier, 1992, tous droits réservés.

L'accès aux archives de la revue «Annales de la faculté des sciences de Toulouse » (http://picard.ups-tlse.fr/ annales/) implique l'accord avec les conditions générales d'utilisation (http://www.numdam.org/conditions). Toute utilisation commerciale ou impression systématique est constitutive d'une infraction pénale. Toute copie ou impression de ce fichier doit contenir la présente mention de copyright.

\section{Numdam}

Article numérisé dans le cadre du programme

Numérisation de documents anciens mathématiques

http://www.numdam.org/ 


\title{
Limiting angle of Brownian motion in certain two-dimensional Cartan-Hadamard manifolds
}

\author{
PeI HsU ${ }^{(1)}$ and Wilfrid S. Kendall ${ }^{(2)}$
}

RÉsumé. - Soit IH une surface du type Cartan-Hadamard dont la courbure admet une borne supérieure négative très faible mais pas de borne inférieure. Nous montrons que lorsque le temps croît vers le temps d'explosion alors le processus angulaire du mouvement brownien riemannien sur $\mathbb{I H}$ tend vers une limite non dégénérée dont la distribution est partout dense sur le cercle à l'infini de la variété $\mathbb{I H}$.

Abstract. - Suppose that $\mathbb{I H}$ is a two-dimensional Cartan-Hadamard manifold with sectional curvatures satisfying a weak negative upper bound and no lower bound. Then the angular part of Brownian motion on $\mathbb{H}$ tends to a limit as time tends to the explosion time of the Brownian motion. Moreover this limit angle has a distribution whose closed support is dense on the circle at infinity.

KEY-WORDS : Brownian motion, Cartan-Hadamard manifold, comparison arguments, geodesic, limiting angle, sectional curvature, stochastic differential equations.

Subject Classification 60J65, 58G32

\section{Introduction}

Recall that a Cartan-Hadamard manifold $\boldsymbol{H}$ is a simply- connected complete Riemannian manifold for which all the sectional curvatures are nonpositive. The theorem of Cartan-Hadamard states that $\mathbb{H}$ is diffeomorphic to Euclidean space, furthermore that this diffeomorphism is realized by the exponential map $\operatorname{Exp}_{\mathbf{o}}: \mathbf{H} \rightarrow \mathbb{R}^{n}$ at any fixed reference point $\mathbf{o} \in \mathbb{H}$.

(1) Department of Mathematics, Northwestern University, Evanston, IL 60208, USA

(2) Department of Statistics, University of Warwick, Coventry CV4 7 AL, UK 
Thus Cartan-Hadamard manifolds allow the existence of global systems of geodesic polar coordinates $(r, \theta)$, where $r(x)=\operatorname{dist}(x, o)$ and $\theta$ is defined by

$$
\operatorname{Exp}_{\mathrm{o}}(r(x), \theta(x))=x \quad \text { for all } x \in \mathbb{H}
$$

(the argument of Exp being given in Euclidean polar coordinates).

We denote Brownian motion on $\mathbb{H}$ by $\mathrm{BM}(\mathbf{H})$, and ordinary real-valued Brownian motion by $\mathrm{BM}(\mathrm{R})$. If we wish to emphasize the starting point we use a suffix; thus $\mathrm{BM}_{0}(\mathbf{R})$ denotes real-valued Brownian motion begun at 0 . Note that $\mathrm{BM}(\mathbb{H})$ has a lifetime $\xi$ (the explosion time) which may be finite (because there is no lower bound on the curvature). We shall use the notation of Kendall [15] for the basic constructs of stochastic differential geometry. See Elworthy [4] or Rogers and Williams [22] for other treatments of stochastic differential geometry.

The purpose of this paper is to establish that if $\mathbb{H}$ is a two-dimensional Cartan-Hadamard manifold with sectional curvatures satisfying a weak negative upper bound then the angular part $\Theta$ of $\operatorname{BM}(\mathbf{H})$ tends to a limit as time tends to the explosion time $\xi$. Moreover this limit angle has a distribution whose closed support is dense on the circle at infinity. This result extends the work of Kendall [11] and itself shows promise of generalizing to higher dimensions at the price of requiring a lower (but generous) bound on the curvatures.

We first give a brief survey of work on the limiting angular behaviour of Brownian motion on Cartan-Hadamard manifolds with at least some upper curvature bound.

Dynkin [3] introduced the problem of studying the asymptotic behaviour of Brownian motion on a negatively curved manifold. Prat [21] established existence of a limiting angle of Brownian motion on Cartan-Hadamard manifolds with constant negative upper and lower curvature bounds. Similar results were obtained at the same time by Kifer [17].

Kendall ([10], announced in [9]) proved limiting angle and density of limiting angle distribution for a class of processes related to Brownian motion (what would now be called $\Gamma$-martingales of $K$-bounded dilatation), living on Cartan-Hadamard manifolds with constant negative upper and lower curvature bounds. These results were used to prove generalized Picard's little theorem for harmonic maps. Goldberg and Mueller [5] generalized this to a case of upper bound on curvature decaying to zero at infinity at an appropriate rate and constant negative lower bound on curvature. 
Greene and $\mathrm{Wu}[6]$ conjectured that a Cartan-Hadamard manifold always possesses nonconstant bounded harmonic functions if outside a fixed compact set its curvature satisfies an upper bound $-c / r(x)^{2}$ decaying proportionally to the inverse of squared geodesic distance from a fixed point $o$. Sullivan [24] used limiting angle and density of angular distribution for Brownian motion to settle the Greene-Wu conjecture for the special case of a Cartan-Hadamard manifold of constant negative upper and lower curvature bounds. (At the same time Anderson [1], proved the same results using analytic methods.)

Kendall [11] proved limiting angle and density of limiting angle distribution for Brownian motion on a two-dimensional Cartan-Hadamard manifold with no lower bound on the curvature and a constant negative upper curvature bound. (Equivalent results were proved using analytic methods in Choi, $[26])$.

Hsu and March [7] proved limiting angle and density of limiting angle distribution for Brownian motion on a Cartan- Hadamard manifold with variable lower and upper negative bounds on curvature. Essentially, the Hsu-March result works for the Greene-Wu upper curvature bound $-c / r(x)^{2}$ when $c=\alpha(\alpha-1)>2$, if the lower bound is $-L^{2} r(x)^{2 \beta}$ for $\beta$ with $\alpha(1-\beta)>2$. (The survey of Kendall [14], gives a statement of the corresponding $\Gamma$-martingale result).

The lower curvature bound is annoying. It occurs in all the cases above except for the two-dimensional case. March [18] discusses the rotationally-symmetric case where the lower bound is not needed. It is natural to wonder whether the intervention of the lower bound is simply due to limitations of present techniques. This appears not to be the case: Ancona (unpublished) has constructed a three- dimensional example of a warped product of Riemannian manifolds, for which the limiting direction of Brownian motion has positive probability of taking one specific value (although it is easily seen that there are many nonconstant bounded harmonic functions corresponding to this single discrete event). It seems plausible that a refinement of this construction would produce cases where the limiting behaviour of the Brownian motion was of spiral form, with no limiting direction at all. In any case this casts strong doubt on the ability of direct angular convergence techniques to resolve the Greene-Wu conjecture, since Ancona's example shows that in the case of uncontrolled negative curvature it is no longer possible to obtain full information about the asymptotics of Brownian motion by means of direct comparison with geodesic rays at fixed angles. 
In this paper we set ourselves the modest target of extending the ideas of Kendall [11] and Hsu-March [7] to deal with the case of limiting angle of Brownian motion on a two-dimensional Cartan-Hadamard manifold with no lower bound on the curvature and an upper curvature bound which is nonconstant, decaying to zero at infinity at an appropriate rate. The proof in Kendall [11] was based on a simple comparison argument using the distance of Brownian motion from a fixed geodesic line - the distance may be bounded below by real-valued Brownian motion plus drift which is bounded away from zero when the distance is bounded away fro:n zero. This simple comparison argument is not powerful enough if the curvature is allowed to decay to zero at infinity, since the argument does not then bound the drift away from zero. It is necessary to employ also wha' might be called the classical argument, which is to say the line of attack used in most of the above references and which appears first in Prat [21].

In the next section, section 2, we establish the fundamental comparison which underlies our arguments here and which also forms the basis of Kendall [11], concerning the distance between Brownian motion and geodesic in a two-dimensional Cartan-Hadamard manifold. The argument in Kendall [11] uses an incorrect formula (though the main lines of the argument are not affected by this). We therefore here give a full proof of a correct version of the formula in question.

In section 3 we establish a sequence of preliminary lemmas which build up to the main result. We state the main result here for ease of reference:

THEOREM (Theorem 3.6 below.). - Let $\mathbb{H}$ be a two-dimensional CartanHadamard manifold with sectional curvatures bounded above by $-c / r^{2}$ for some $c>0$ off a compact set, and let $X$ be $\mathbf{B M}(\mathbf{H})$. Then $\Theta=\theta(X)$ converges to a limit $\Theta(\infty)$ as time tends to the explosion time $\xi$. Furthermore the distribution of $\Theta(\infty)$ has as closed support the entire circle $S^{1}$ of directions.

From this result there follows immediately as a corollary the full Greene$\mathrm{Wu}$ conjecture in the two-dimensional case. Of course this corollary is of no particular interest since stronger results follow from complex analysis (Greene and Wu [6], Proposition 7.5). However the result does give us a probabilistic way of constructing nonconstant bounded harmonic functions, and further information on the probabilistic effects of negative curvature in the two-dimensional case. Note also that our Theorem 3.6 give specific information about the asymptotic angular behaviour of $\mathrm{BM}(\mathbb{H})$, and this information is not available from complex analysis methods. 
The approach used in this paper has some potential for extension to higher dimensions, although there are several technicalities which must be overcome. We hope to explore this at a later date. We discuss this possibility, and also the essential limitations of the approach, in the concluding section of this paper.

The first-named author gratefully acknowledges support from an EC twinning grant, and the hospitality of the Mathematics Institute of the University of Warwick, over the period during which this work was carried out.

\section{The distance of Brownian motion from a geodesic}

Kendall [11] used a stochastic analysis of the distance of Brownian motion from a geodesic to show that the limiting angle exists for Brownian motion on a two-dimensional Cartan-Hadamard manifold with curvatures bounded above by a strictly negative constant. Unfortunately the formula (3.2) given there is wrong, both because a scaling argument is applied wrongly and also because it uses a formula for the Laplacian of this distance (Greene and Wu [6], page 117) which appears to be incorrect. The conclusions of Kendall [11] remain correct since they depend only on the general form of the analysis. We shall need this analysis in Section 3 and therefore we give a corrected treatment here.

Theorem 2.1. - Let $\mathbf{H}$ be a two-dimensional Cartan-Hadamard manifold, and suppose the sectional curvatures in $\mathbb{H}$ are everywhere bounded above by a non-positive constant (possibly zero) $-H^{2} \leq 0$. Let $X$ be $B M(\mathbb{H})$. Let $\gamma: \mathbf{R} \rightarrow \mathbb{H}$ be a geodesic line and define

$$
\tilde{R}(t)=\operatorname{dist}(X(t), \operatorname{Im} \gamma) .
$$

Then there is a $\mathbf{B M}_{0}(\mathbf{R})$, namely $\tilde{B}$, (perhaps stopped at a Markov random time) such that

$$
\tilde{R}(t) \geq \tilde{R}(0)+\tilde{B}(t)+\frac{1}{2} \int_{0}^{t} H \tanh (H \tilde{R}(s)) \mathrm{d} s
$$

up to the first time that $\tilde{R}$ vanishes.

Remark. - Only the case $H=0$ is important for the work below, in contrast to Kendall [11] which requires the case $H>0$. 
Proof. - This follows from the geometric form of Itô's lemma (see for example Kendall [15], Theorem 2) and estimates on Jacobi vectorfields following from comparison arguments. Let $\Xi$ and $M$ be the stochastic parallel transport and development for $X$ (so in particular $M$ is a Euclidean Brownian motion, stopped at the explosion time $\xi$ of $X$ if that is finite). The geometric form of Itô's lemma shows that for any $C^{2}$ function $f: \mathbf{H} \rightarrow \mathbb{R}$ we have

$$
\mathrm{d}_{I} f(X)=[\operatorname{grad} f(X) \cdot \Xi] \mathrm{d}_{I} M+\frac{1}{2} \operatorname{trace}(\text { Hess } f(X) \cdot(\Xi, \Xi) \cdot \mathrm{d}[M, M])
$$

(where $\mathrm{d}_{I} f(X), \mathrm{d}_{I} M$ are Itô differentials).

We may apply the above to $f: y \mapsto \operatorname{dist}(y, \operatorname{Im} \gamma)$ so long as we stop the resulting stochastic differential equation as soon as smoothness fails (precisely when $\tilde{R}$ hits 0 ). The triangle inequality and differentiation along the minimal geodesic from $x$ to $\operatorname{Im} \gamma$ together show $\|\operatorname{grad} f\|=1$ on $\mathbb{H} \backslash \operatorname{Im} \gamma$. Hence the stochastic differential $[\operatorname{grad} f(X) \cdot \Xi] \mathrm{d}_{I} M$ integrates to yield a real-valued Brownian motion $\tilde{B}$ begun at 0 , stopped at the Markov time $\xi$ if it is finite.

On the other hand $\mathrm{d}[M, M]$ is diagonal in form, with diagonal entries of $\mathrm{d} t$. Hence the second term reduces:

$$
\frac{1}{2} \operatorname{trace}(\operatorname{Hess} f(X) \cdot(\Xi, \Xi) \cdot \mathrm{d}[M, M])=\frac{1}{2} \Delta f(X) \mathrm{d} t .
$$

Now in this two-dimensional case

$$
\Delta f(x)=\left[\frac{\mathrm{d}^{2} f}{\mathrm{~d} u^{2}}(\lambda(u))\right]_{u=0}
$$

where $\lambda$ is a unit-speed geodesic begun at $x$ and with initial velocity $\lambda^{\prime}(0)$ normal to the minimal geodesic from $x$ to $\operatorname{Im} \gamma$. Working with the appropriate geodesic rectangle we may deduce

$$
\left[\frac{\mathrm{d}^{2} f}{\mathrm{~d} u^{2}}(\lambda(u))\right]_{u=0}=\left[\left\langle J^{\prime}, J\right\rangle\right]_{s=0}^{s=f(x)} .
$$

Here $J=\{J(s): s \in[0, f(x)]\}$ is the Jacobi vectorfield running along the minimal geodesic from $x$ to $\operatorname{Im} \gamma$, with $J(0)=\lambda^{\prime}(0)$ and $\left[J^{\prime}\right]_{s=f(x)}=0$. Note that $J(s)$ is located at distance $s$ from $x$. Comparison arguments for Jacobi vectorfields (following Cheeger and Ebin [2], Lemma 1.22) show that

$$
\left[\left\langle J^{\prime}, J\right\rangle\right]_{s=0}^{s=f(x)} \geq\left[\tilde{J}^{\prime} \cdot \tilde{J}\right]_{s=0}^{s=f(x)}
$$


where the function $\tilde{J}$ is derived from the corresponding Jacobi vectorfield in the case of constant curvature $-H^{2}$ :

$$
\tilde{J}(s)=\frac{\cosh (H(f(x)-s))}{\cosh (H f(x))} .
$$

Hence

$$
\Delta f(x) \geq H \tanh (H f(x)) .
$$

The result follows from the usual comparison theorem for stochastic differential equations (Yamada [25]).

Remark. - Note that $\tilde{B}$ is identified in the course of the proof as arising from integration of the gradient composed with the parallel transport against the stopped Euclidean Brownian motion which is the stochastic development. Hence $\tilde{B}$ is adapted to the intrinsic filtration of $X$.

Remark. - A similar result can be proved for $\Gamma$-martingales in $\mathbf{H}$, except that $\tilde{B}$ must be replaced by a continuous real-valued local martingale and the $d s$ term in the integral must be replaced by a component of the stochastic parallel transport applied to the differential of the quadratic variation of the stochastic development. In order to obtain useful results in such cases one requires bounds on the "dilatation" of the $\Gamma$-martingale, after Kendall ([9], $[10],[12])$.

Remark. - There are obvious higher-dimensional generalizations; for example the case of the distance of a Brownian motion from a totally geodesic hypersurface in a Cartan-Hadamard manifold. However, in contrast to the two-dimensional case, totally-geodesic hypersurfaces are in short supply in general higher-dimensional manifolds. It may be possible to exploit generalizations to distances from geodesically convex hypersurfaces, and we hope to return to this at a later time.

We now state a corollary which is the special case of the above which we will use in the next section.

Corollary 2.2. - Let $\mathbb{H}$ be any two-dimensional Cartan-Hadamard manifold and let $X$ be $\mathbf{B M}(\mathbf{H})$. Given a geodesic $\gamma: \mathbb{R} \rightarrow \mathbb{H}$ there exists $\tilde{B}$, $a \mathbf{B M}_{0}(\mathbf{R})$ adapted to the filtration of $X$, such that

$$
\operatorname{dist}(X, \operatorname{Im} \gamma) \geq \tilde{B}+\operatorname{dist}\left(X_{0}, \operatorname{Im} \gamma\right)
$$

up to the first time which is a zero of $\operatorname{dist}(X, \operatorname{Im} \gamma)$. 


\section{The limiting angle}

In the following $\mathbb{H}$ will be a two-dimensional Cartan-Hadamard manifold with curvature function $-\kappa^{2}: \mathbb{H} \rightarrow(-\infty, 0]$ satisfying the upper bound

$$
-\kappa^{2}(x) \leq-\frac{c}{\operatorname{dist}(x, o)^{2}} \quad \text { for all } x \in \mathbb{H} \text { off a fixed compact set } \mathcal{K}
$$

for some constant $c>0$ and some fixed reference point $o \in \mathbb{H}$. It is convenient to define the associated constant $\alpha>1$ by

$$
c=\alpha(\alpha-1)
$$

We set $\mathcal{R}=\sup \{\operatorname{dist}(x, o): x \in \mathcal{K}\}$.

Let $X$ be $\operatorname{BM}(\mathbf{H})$. The main result of this paper concerns the asymptotic behaviour of the angular process $\Theta=\theta(X)$. We shall show (Theorem 3.6 below) that as time tends to the explosion time $\xi$ so the angular process $\Theta$ converges to a limiting direction.

Before stating and proving our Theorem we review some well-known geometric comparison results and prove a number of preliminary lemmas.

Suppose the metric on $\mathbf{H}$ is given in geodesic polar coordinates based at o by the formula

$$
\mathrm{d} s^{2}=\mathrm{d} r \otimes \mathrm{d} r+g(x)^{2} \mathrm{~d} \theta \otimes \mathrm{d} \theta .
$$

Then $g$ solves the Jacobi equation: its second radial derivative is given by

$$
g_{r r}=\frac{\partial^{2} g}{\partial r^{2}}=\kappa^{2}(x) \cdot g
$$

with initial conditions $g(\mathbf{o})=0$ and $\lim _{r \rightarrow 0} g_{r}(r, \theta)=1$ for all $\theta$.

Comparison arguments for second-order ordinary differential equations, which we sketch here, then show that

(i) there is a constant $C_{1}>0$ (depending on $\mathcal{K}$ ) such that for all points $y$

$$
g(y) \geq C_{1} \operatorname{dist}(y, \mathbf{o})^{\alpha} .
$$

This follows by comparison of $g$ with $\tilde{g}(r, \theta)=C_{1} r^{\alpha}$. The Jacobi equation for $g$ is nonconjugate (since the curvature is nonpositive everywhere) and so $g_{r}$ never vanishes. Hence we may choose $C_{1}$ 
Limiting angle of Brownian motion in two-dimensional Cartan-Hadamard

so that $g>\tilde{g}, g_{r}>\tilde{g}_{r}$ whenever $r=\mathcal{R}$. For $r>\mathcal{R}$ we find $\tilde{g}_{r r}=\alpha(\alpha-1) \tilde{g} / r$, and note that $\kappa^{2}(x) \geq \alpha(\alpha-1) / r$. So we may apply a comparison theorem found in Milnor [19] (see also Greene and $\mathrm{Wu}[6]$, Lemma 2.31) to deduce the required inequality.

(ii) for all $r$ we have

$$
g_{r} / g \geq b(r)
$$

where $b(r)$ is given by replacing $g_{r} / g$ by the corresponding expression $\hat{g}_{r} / \hat{g}$ for the explicit solution $\hat{g}(r)$ of the Jacobi equation with $\kappa^{2}(x)$ replaced by 0 for $r(x)<\mathcal{R}$ and by $c / r(x)^{2}$ for $r(x) \geq \mathcal{R}$ and initial conditions $\hat{g}(0)=0, \hat{g}_{r}(0)=1$;

$$
b(r)= \begin{cases}1 / r & \text { if } r<\mathcal{R} \\ \alpha / r-(2 \alpha-1) /\left(r+(\alpha \mathcal{R} /(\alpha-1))(r / \mathcal{R})^{2 \alpha}\right) & \text { if } r \geq \mathcal{R}\end{cases}
$$

and hence for each $\eta>0$ there is $\rho_{\eta}>\mathcal{R}$ such that for all $r>\rho_{\eta}$ we have

$$
g_{r} / g \geq \frac{\alpha-\eta}{r}
$$

Essentially this follows from Milnor's comparison result given above, applied to the Jacobi differential equations governing

$$
f(u)=\frac{g(r-u)}{g(r)} \text { and } \hat{f}(u)=\frac{\hat{g}(r-u)}{\hat{g}(r)} \text { over } u \in[0, r] .
$$

Because of nonconjugacy it would follow from $f^{\prime}(0)<\hat{f}^{\prime}(0)$ that $f(r)<\hat{f}(r)$. But we know $f(r)=0=\hat{f}(r)$.

The first lemma is a stochastic differential equation comparison result for the radial process $R=r(X)$.

LemmA 3.1. - There exists a $\mathbf{B M}_{0}(\mathbf{R}), B$, possibly stopped at the explosion time of $X$, and in any case adapted to the filtration of $X$, such that

$$
R(t) \geq R(0)+B(t)+\frac{1}{2} \int_{0}^{t} b(R(s)) \mathrm{d} s
$$

where $b$ is the function given above. 
Proof.- The Ito stochastic differential equation for $R$ is

$$
\mathrm{d}_{I} R=\mathrm{d}_{I} B+\frac{1}{2}\left(\frac{g_{r}(X)}{g(X)}\right) \mathrm{d} t
$$

where $B$ is $\mathbf{B M}_{0}(\mathbf{R})$, stopped at the explosion time of $X$ when this is finite, and adapted to the natural filtration of $X$. This follows from the same argument used to analyse $\operatorname{dist}(X, \operatorname{Im} \gamma)$ in the proof of Theorem 2.1 above, and the facts that grad $r=1$ and that $\Delta r=g_{r} / g$. The result follows from the bound above on $g_{r} / g$ and the comparison argument of Yamada [25].

The Bessel process $Y$ of index $\alpha$ solves the stochastic differential equation

$$
\mathrm{d} Y=\mathrm{d} B+\frac{\alpha}{2 Y} \mathrm{~d} t
$$

for $B$ a $\operatorname{BM}(\mathbf{R})$. If $\alpha$ is a nonnegative integer then $Y$ arises as the radial part of Brownian motion in $(1+\alpha)$-dimensional Euclidean space. If $\alpha>1$ then $Y$ is transient. It follows that $R \rightarrow \infty$ as time tends to infinity (under the convention that $R$ is frozen at $\infty$ after the explosion time). For each time $R$ reaches a high enough level it has a positive chance of escaping to infinity, by comparison with a Bessel process of index just less than $\alpha$. In the following $\mathrm{BES}_{r}^{\alpha}$ will denote a Bessel process of index $\alpha$ started at level $r$. Let $P$ denote the probability measure for $\mathrm{BES}_{r}^{\alpha}$ (information about the index $\alpha$ and the starting level $r$ being carried in the notation $\mathrm{BES}_{r}^{\alpha}$ ).

We formalize this comparison in a lemma. Let $\mathbf{P}^{r, \theta}$ abbreviate the conditional probability measure obtained from $\mathbf{P}$ ( where $\mathbf{P}$ is the probability measure for the Riemannian Brownian motion $X$ with some random starting point with probability density positive over the whole manifold) by conditioning on $\bar{K}(0)=r, \Theta(0)=\theta$.

LEMma 3.2. - For each $\epsilon>0$ such that $\alpha-\epsilon>1$ there is a $\mathrm{BES}_{r}^{\alpha-\epsilon}$, which can be defined on and adapted to the same probability space as $X$, such that for all $r \geq \rho_{\epsilon}$

$$
\mathbf{P}^{r, \theta}\left[R(t) \geq \operatorname{BES}_{r}^{\alpha-\epsilon}(t) \text { for all } t\right] \geq 1-\left(\frac{\rho_{\epsilon}}{r}\right)^{\alpha-\epsilon-1} .
$$

Proof. - This follows from the comparison result in lemma 3.1, a further comparison with the stochastic differential equation for $\mathrm{BES}_{r}^{\alpha-\epsilon}$ using Yamada [25], and calculation of the probability of $\mathrm{BES}_{r}^{\alpha-\epsilon}$ hitting the level $\rho_{\epsilon}$. 
We exploit this comparison using a result stated in Shiga and Watanabe [23] and essentially due to Motoo [20]; since the Bessel process of dimension higher than 2 escapes to infinity at a rate which may be estimated, we may obtain a lower bound on the rate of escape for the radial process $R$ as well.

Lemma 3.3. - For all sufficiently small positive $\eta$, and for each $\epsilon$ such that $\alpha-\epsilon>1$,

$$
\mathbf{P}^{r, \theta}\left[R(t) \geq \sqrt{r} \vee t^{\frac{1}{2}-\eta} \text { for all } t\right] \geq p(r)
$$

where

$$
\begin{aligned}
1-p(r)= & r^{-(\alpha-\epsilon-1) / 2}+\left(\frac{\rho_{\epsilon}}{r}\right)^{\alpha-\epsilon-1}+ \\
& +P\left[\mathrm{BES}_{0}^{\alpha-\epsilon}<t^{\frac{1}{2}-\eta} \text { for some } t \geq r^{1 /(1-2 \eta)}\right] .
\end{aligned}
$$

Note that the probability on the left-hand side of the bound therefore converges to 1 uniformly in $\theta$ as $r$ tends to $\infty$.

Proof.- Because of lemma 3.2 it suffices to work with $\mathrm{BES}_{r}^{\alpha-\epsilon}$ rather than $R$.

Theorem 3.2 part (i) of Shiga and Watanabe [23], attributed there to Motoo [20], shows that almost surely for any such Bessel process BES ${ }^{\alpha-\epsilon}$

$$
\operatorname{BES}_{1}^{\alpha-\epsilon}(t) \geq t^{\frac{1}{2}-\eta} \quad \text { for all sufficiently large } t .
$$

Moreover

$$
P\left[\operatorname{BES}_{r}^{\alpha-\epsilon}(t) \geq \sqrt{r} \text { for all } t\right]=1-r^{-(\alpha-\epsilon-1) / 2} .
$$

Comparison of $\mathrm{BES}_{r}^{\alpha-\epsilon}$ with $\mathrm{BES}_{1}^{\alpha-\epsilon}$ now shows

$$
\begin{aligned}
1-P & {\left[\operatorname{BES}_{r}^{\alpha-\epsilon}(t) \geq\left(\sqrt{r} \vee t^{\frac{1}{2}-\eta} \text { for all } t\right] \leq\right.} \\
& \leq r^{-(\alpha-\epsilon-1) / 2}+P\left[\operatorname{BES}_{1}^{\alpha-\epsilon}(t)<t^{\frac{1}{2}-\eta} \text { for some } t \geq r^{1 /(1-2 \eta)}\right]
\end{aligned}
$$

which is as required because of Lemma 3.2.

Remark. - An alternative proof of the special case of the Motoo-ShigaWatanabe result required above is given in Kendall [16], using the computer algebra methods of symbolic Itô calculus. 
The next lemma is an adaptation of Hsu and March ([7], lemma 2) to suit the concerns of this paper.

Lemma 3.4. - Fix $x \in \mathbb{H} \backslash\{\mathbf{o}\}$ and $\epsilon>\mathbf{0}$. Let $\boldsymbol{\gamma}$ be the geodesic through o in $\mathbb{H}$ such that $\gamma^{\prime}(0)$ is at the angle $\theta(x)+\epsilon$. Suppose $r(x)>\mathcal{R}+\operatorname{dist}(x, \operatorname{Im} \gamma)$. Then

$$
\epsilon \leq \frac{\operatorname{dist}(x, \operatorname{Im} \gamma)}{C_{1} \cdot[r(x)-\operatorname{dist}(x, \operatorname{Im} \gamma)]^{\alpha}}
$$

which provides a bound on $\epsilon$ in terms of $r(x)$ and $\operatorname{dist}(x, \operatorname{Im} \gamma)$.

Proof.- Observe

$$
\operatorname{dist}(x, \operatorname{Im} \gamma)=\int_{\theta(x)}^{\theta(x)+\epsilon} \sqrt{\left[\left(\frac{\mathrm{d} r}{\mathrm{~d} \theta}\right)^{2}+g^{2}\right]} \mathrm{d} \theta
$$

where the integration is carried out along the minimal geodesic running from $x$ to $\operatorname{Im} \gamma$. Hence we can use the non-decreasing property of $g$;

$$
\operatorname{dist}(x, \operatorname{Im} \gamma) \geq \int_{\theta(x)}^{\theta(x)+\epsilon} g \mathrm{~d} \theta \geq \epsilon \cdot g(y)
$$

where $y$ is the point on $\operatorname{Im} \gamma$ closest to $x$. For by the negative curvature of $\mathbb{H}$ it follows that $y$ is the point closest to o on the minimal geodesic from $x$ to $\operatorname{Im} \gamma$. The proof is completed by use of the triangle inequality $r(y) \geq r(x)-\operatorname{dist}(x, \operatorname{Im} \gamma) \geq \mathcal{R}$, and use of the bound on $g$ when $r>\mathcal{R}$.

The next lemma is the last preliminary result, and deals with the main technical issues of this paper. First we introduce some notation. Let $\gamma^{\theta}$ be the geodesic through o with angle $\theta$.

Consider the following sequence of stopping times $T_{0}, T_{1}, \ldots$, which measure intervals of time $\left[T_{n}, T_{n+1}\right]$ over which the angle changes by progressively smaller quantities bounded by the random intervals $\left[-\epsilon_{n}^{-}, \epsilon_{n}^{+}\right]$:

$$
\begin{aligned}
T_{0} & =0 \\
\epsilon_{n}^{ \pm} & =\inf \left\{\epsilon>0: X\left(T_{n}\right) \text { is at distance at least } \frac{1}{2} R\left(T_{n}\right) \text { from } \gamma^{\Theta\left(T_{n}\right) \pm \epsilon}\right\} \\
T_{n+1} & =\inf \left\{t>T_{n}: \Theta(t)-\Theta\left(T_{n}\right) \text { is equal to one of } \pm \epsilon_{n}^{ \pm}\right\} .
\end{aligned}
$$

Here $\frac{1}{2}$ may be replaced by any number lying in $(0,1)$. Note that $\epsilon_{n}^{ \pm}$are $\mathcal{F}_{T_{n}}$ measurable random variables. If $T_{n}=\infty$ then we set $\epsilon_{n}^{ \pm}=\epsilon_{n+1}^{ \pm}=\cdots=0$. Set $\epsilon_{n}=\max \left\{\epsilon_{n}^{+}, \epsilon_{n}^{-}\right\}$(and note $\epsilon_{n}>0$ if $T_{n}<\infty$ ). 
The next lemma concerns the convergence of the sum $\sum \epsilon_{n}$.

Lemma 3.5. - For any $r$ and $\theta$ the sum $\sum \epsilon_{n}$ converges with probability 1 under $\mathbf{P}^{r, \theta}$. Moreover, for any $\lambda>0$ we have

$$
\sup _{\theta} \mathbf{P}^{r, \theta}\left[\sum \epsilon_{n} \geq \lambda\right] \rightarrow 0
$$

as $r \rightarrow \infty$.

Proof.- Suppose $T_{n}$ is finite. Consider the time increment $L_{n+1}=$ $T_{n+1}-T_{n}$. By Theorem 2.1 this time increment is bounded below by the minimum of two random times which are the times taken by two different (co-adapted, but not independent) Brownian motions to attain the level $\frac{1}{2} R\left(T_{n}\right)$. The Brownian motions in question are the increments from time $T_{n}$ of the martingale parts of the distance processes $\operatorname{dist}\left(X, \operatorname{Im} \gamma^{\Theta\left(T_{n}\right) \pm \epsilon^{ \pm}}\right)$.

By the strong Markov property these two random times are independent of $\mathcal{F}_{T_{n}}$ conditional on $R\left(T_{n}\right)$, and under this conditioning have distributions which are first passage times of real-valued Brownian motion to the level $\frac{1}{2} R\left(T_{n}\right)$. They are not independent (in cases of strong negative curvature they will indeed be very strongly related) but the simplest estimate of the distribution of their minimum, and hence of $L_{n}$, will suffice:

$$
\begin{aligned}
\mathbf{P}\left\{L_{n+1}>\frac{1}{4} R\left(T_{n}\right)^{2} a \mid \mathcal{F}_{T_{n}}, R\left(T_{n}\right)=r\right\} \geq \\
\geq 1-2 \cdot \mathbf{P}\left\{\tau_{r / 2}\left(\mathbf{B M}_{0}(\mathbb{R})\right)<\frac{1}{4} r^{2} a\right\}= \\
=1-2 \cdot \mathbf{P}\left\{\tau_{1}\left(\mathbf{B M}_{0}(\mathbf{R})\right)<a\right\}
\end{aligned}
$$

where $\tau_{k}\left(\mathrm{BM}_{0}(\mathbf{R})\right)$ is the first passage time of $\mathrm{BM}_{0}(\mathbf{R})$ to the level $k$ and the last step uses Brownian scaling. (Note that the event $\left\{R\left(T_{n}\right)=r\right\}$ is $\mathcal{F}_{T_{n}}$-measurable, and is included in the conditioning above simply to allow us to replace $R\left(T_{n}\right)$ by $r$.)

Fix $\ell>0$ and choose $a$ so that

$$
\ell=1-2 \cdot \mathbf{P}\left\{\tau_{1}\left(\mathbf{B M}_{0}(\mathbf{R})\right)<a\right\}
$$

(thus making the lower bound a positive constant). It follows that a strong law of large numbers may be applied to the sequence of events

$$
\left\{L_{n+1}>\frac{1}{4} R\left(T_{n}\right)^{2} a\right\} .
$$

We give a coupling argument for this. 
Augment the underlying probability space by constructing $V_{1}, V_{2}, \ldots$ to be independent, identically distributed random variables, with uniform distribution on the range $[0,1]$, and such that the whole sequence of new random variables is independent of $\mathcal{F}_{\infty}=\bigvee_{n} \mathcal{F}_{T_{n}}$. Set

$$
H_{n}=\mathbf{P}\left\{L_{n+1}>\frac{1}{4} R\left(T_{n}\right)^{2} a \mid \mathcal{F}_{T_{n}}\right\}
$$

and

$$
U_{n+1}= \begin{cases}1 & \text { if } L_{n+1}>\frac{1}{4} R\left(T_{n}\right)^{2} a \text { and } H_{n} V_{n+1}<\ell \\ 0 & \text { otherwise. }\end{cases}
$$

Then $U_{n+1}$ takes values 0 and 1 and is measurable with respect to the $\sigma$-field generated by $\mathcal{F}_{T_{n+1}}$ and $V_{1}, \ldots, V_{n+1}$. Furthermore

$$
\begin{aligned}
& \mathbf{P}\left\{U_{n+1}=1 \mid \mathcal{F}_{T_{n}}, V_{1}, \ldots, V_{n}\right\}= \\
& \quad=\mathbf{P}\left\{L_{n+1}>\frac{1}{4} R\left(T_{n}\right)^{2} a, H_{n} V_{n+1}<\ell \mid \mathcal{F}_{T_{n}}, V_{1}, \ldots, V_{i 2}\right\} \\
& =\frac{\ell}{H_{n}} \cdot \mathbf{P}\left\{L_{n+1}>\frac{1}{4} R\left(T_{n}\right)^{2} a \mid \mathcal{F}_{T_{n}}, V_{1}, \ldots, V_{n}\right\} \\
& =\frac{\ell}{H_{n}} \cdot \mathbf{P}\left\{L_{n+1}>\frac{1}{4} R\left(T_{n}\right)^{2} a \mid \mathcal{F}_{T_{n}}\right\}=\ell
\end{aligned}
$$

where the second equality follows from the fact that $V_{n+1}$ is independent of the other quantities involved, while the third equality follows from the fact that $V_{1}, \ldots, V_{n}$ are independent of $\mathcal{F}_{T_{n+1}}$.

Thus the $U_{n+1}$ form an independent and identically distributed sequence of nondegenerate $\{0,1\}$ valued random variables and, by construction, $L_{n+1}>\frac{1}{4} R\left(T_{n}\right)^{2} a U_{n+1}$. We may now employ the information we have on the growth rate of $R$.

Lemma 3.3 shows that at sufficiently large times $R$ is bounded below by a power of time just less than square-root with high probability. To be precise, for any sufficiently small positive $\eta$,

$$
\mathbf{P}^{r, \theta}\left[R\left(T_{n}\right) \geq\left(\sqrt{r} \vee T_{n}^{\frac{1}{2}-\eta}\right) \text { for all } n\right] \geq p(r) .
$$

Now

$$
T_{n+1}-T_{n}=L_{n+1}>\frac{1}{4} a R\left(T_{n}\right)^{2} \cdot U_{n+1}
$$


Limiting angle of Brownian motion in two-dimensional Cartan-Hadamard

and so with $\mathbf{P}^{r, \theta}$-probability at least $p(r)$

$$
\frac{T_{n+1}-T_{n}}{T_{n}^{1-2 \eta}} \geq \frac{1}{4} a U_{n+1} .
$$

Thus by induction, for some constant $C_{2}$ depending on $a, \ell$, and $\eta$, and with $\mathbf{P}^{(r, \theta)}$-probability at least $p(r)$,

$$
T_{n} \geq C_{2} S_{n}^{\frac{1}{2 \eta}}
$$

where

$$
S_{n}=\sum_{k=1}^{n} U_{k}
$$

Now by lemma 3.4 ,

$$
R\left(T_{n}\right) \geq 3 \mathcal{R} / 2 \quad \text { implies } \quad \epsilon_{n} \leq C_{3} R\left(T_{n}\right)^{1-\alpha}
$$

By lemma 3.3 we see that if $r \geq(3 \mathcal{R} / 2)^{2}$ then with probability at least $p(r)$,

$$
\epsilon_{n} \leq C_{4}\left[\sqrt{r} \vee T_{n}^{1 / 2-\eta}\right]^{1-\alpha} \quad \text { for all } n
$$

Using the lower bound of $T_{n}$ given above we conclude that with probability at least $p(r)$,

$$
\sum \epsilon_{n} \leq C_{5} \sum_{n=1}^{\infty}\left[\sqrt{r} \vee S_{n}^{(1-2 \eta) / 4 \eta}\right]^{1-\alpha}
$$

(recall $\alpha>1$ ). The conclusion of the Lemma follows directly from the law of large numbers and the fact that $p(r) \rightarrow 1$ as $r \rightarrow \infty$.

We now state and prove the main theorem of this paper.

THEOREM 3.6. - Limiting angle of $\mathrm{BM}(\mathbf{H})$. Let $\mathbf{H}$ be as above and let $X$ be $B M(\mathbf{H})$. Then $\Theta=\theta(X)$ converges to a limit $\Theta(\infty)$ as time tends to the explosion time $\xi$. Furthermore the distribution of $\Theta(\infty)$ has as closed support the entire circle $S^{1}$ of directions.

Proof.- This follows immediately from Lemma 3.5 above, since the sum $\sum \epsilon_{n}$ bounds the oscillation of the process $\Theta(t)-\Theta(0)$. 


\section{Conclusion}

It is natural to ask whether these methods can be extended to cover Proposition 7.5 of Greene and $\mathrm{Wu}$ [6], which says that if the upper curvature bound for two-dimensional $\mathbb{H}$ is

$$
-\frac{1+\epsilon}{r^{2} \log r} \quad \text { for } \epsilon>0
$$

off a compact set then $\mathbb{H}$ has the complex structure of the unit disc. This is possible in the rotationally symmetric case (see March [18]). However the methods given above do appear to depend on having curvature decay at most inverse quadratic in the radial distance - we can make available to the interested enquirer an argument which indicates this.

Generalizations to higher dimensions must deal with the technical difficulties of applying comparison arguments not to geodesics but to geodesic cones, with consequent problems about loci of focal points. We think that this can be dealt with, at the price of introducing a lower curvature bound, and we believe that the ensuing results will improve on those of Hsu and March [7]; we hope to return to this point in a later paper.

However this approach, and any others which in the end depend on comparisons of Brownian motion with radial geodesic processes, is unlikely to provide a final answer to the Greene-Wu conjecture mentioned in the introduction. Ancona's counterexample makes this clear; take a rotationallysymmetric and stochastically explosive Cartan-Hadamar manifold $\mathbf{H}$ with constant negative upper bound on its negative curvature and consider the warped metric product $\mathbb{H} \times(0, \infty)$ parametrized by $(h, y)$ and using the metric

$$
\mathrm{d} s^{2}=\frac{g_{\mathrm{H}}+\mathrm{d} y \otimes \mathrm{d} y}{y^{2}}
$$

where $g_{\mathbb{H}}$ is the metric for $\mathbb{H}$. It can be shown that

(i) the product manifold is Cartan-Hadamard, with constant negative upper bound on its negative curvature,

(ii) with positive probability, the limiting angle of its Brownian motion is the angle pointing up the positive $y$-axis,

(iii) but in fact nonconstant bounded harmonic functions may be constructed using the limiting angle obtained by projection onto the $\mathbb{H}$ manifold. 
It is clear that direct angular comparison with geodesic rays is no longer sufficient to extract all the information about asymptotic behaviour. It therefore seems that a final answer to the Greene-Wu conjecture must somehow exploit comparison of Brownian motion with itself, rather than with geodesic rays as has been the practice hitherto. The ideas of Kendall ([12], [13]) may prove helpful here.

\section{References}

[1] Anderson (M.T.) . - The Dirichlet Problem at Infinity for Manifolds of Negative Curvature,

J. Diff. Geom. 18 (1983), pp. 701-722.

[2] Cheeger (J.) and Ebin (D.G.) .- Comparison Theorems in Riemannian Geometry, North Holland, Amsterdam (1975).

[3] Dynkin (E.B.) .- Markov Processes, Springer-Verlag, Berlin (1965).

[4] Elworthy (K.D.) . - Stochastic Differential Equations on Manifolds, Cambridge University Press, Cambridge (1982).

[5] Goldberg (S.I.) and Mueller (C.) . - Brownian motion, geometry, and generalizations of Picard's little theorem, Ann. Probab. 11 (1983), pp. 833-846.

[6] Greene (R.E.) and WU (H.) . - Function Theory on Manifolds Which Possess a Pole, Springer Lecture Notes in Mathematics 699, Springer-Verlag, Berlin (1979).

[7] Hsu (P.) and MARCH (P.) . - The Limiting Angle of Certain Riemannian Brownian Motions,

Comm. Pure and Applied Math. 38 (1985), pp. 755-768.

[8] Huang (H.) and Kendall (W.S.) .- Correction note to "Martingales on Manifolds and Harmonic Maps",

In: Stochastics 37 (1991), pp. 253-257.

[9] Kendall (W.S.) .- Brownian motion, negative curvature, and harmonic maps, In: Stochastic Integrals, Proceedings, London Mathematical Society Durham Symposium, 1980, (ed. D. Williams), Springer Lecture Notes in Mathematics 851 Springer, Berlin (1981), pp. 479-491.

[10] Kendall (W.S.) .- Brownian motion and a generalised little Picard's theorem, Trans. Amer. Math. Soc. 275 (1983), pp. 751-760.

[11] KendalL (W.S.) . - Brownian motion on 2-dimensional manifolds of negative curvature,

Séminaire de Probabilités XVIII, Springer Lecture Notes in Mathematics 1059, Springer-Verlag, Berlin (1984), pp. 70-76.

[12] Kendall (W.S.) .- Stochastic differential geometry, a coupling property, and harmonic maps,

Journal London Math. Soc. 33 (1986a), pp. 554-566. 
[13] Kendall (W.S.) . - Nonnegative Ricci curvature and the Brownian coupling property,

Stochastics 19 (1986b), pp. 111-129.

[14] Kendall (W.S.) . - Stochastic differential geometry,

In: Proceeding of the First World Congress of the Bernoulli Society (eds.

Yu.V. Prohorov and V.V. Sazonov), volume 1, VNU Press, Utrecht (1987), pp. 515-524.

[15] Kendall (W.S.) - - Martingales on Manifolds and Harmonic Maps, Contemporary Mathematics 73 (1988), pp. 121-157; see also Huang and Kendall (1991).

[16] Kendall (W.S.) . - Symbolic Itô calculus: an introduction,

Department of Statistics, University of Warwick, Research Report 217 (1991).

[17] KIFER (J.) . - Brownian motion and harmonic functions on manifolds of negative curvature, Theor. Probab. Appl. 21 (1976), pp. 81-95.

[18] MARCH (P.) . - Brownian motion and harmonic functions on rotationally symmetric manifolds, Ann. Probab. 14 (1986), pp. 793-801.

[19] MilnoR (J.) .- On deciding whether a surface is parabolic or hyperbolic, Amer. Math. Monthly 84 (1977), pp. 43-46.

[20] Мотоо (M.). - Proof of the law of iterated logarithm through diffusion equation, Ann. Inst. Statist. Math. 10 (1959), pp. 21-28.

[21] Prat (J.J.) . - Étude Asymptotique et Convergence Angulaire du Mouvement Brownien sur une Variété à Courbure Négative,

C. R. Acad. Sci. A280 (1975), pp. 1539-1542.

[22] Rogers (L.C.G.) and Williams (D.) .- Diffusions, Markov Processes, and Martingales,

Volume 2, Wiley, Chichester (1987).

[23] Shiga (T.) and Watanabe (S.). - Bessel Diffusions as a One-Parameter Family of Diffusion Processes,

Zeitschrift für Wahrscheinlichkeitstheorie und v. Geb. 27 (1973), pp. 37-46.

[24] Sullivan (D.) . - The Dirichlet Problem at Infinity for a Negatively Curved Manifold,

J. Diff. Geom. 18 (1983), pp. 723-732.

[25] YAMADA (Y.).- On a comparison theorem for solutions of stochastic differential equations and its applications, J. Math. Kyoto Univ. 13 (1973), pp. 497-512.

[26] Chor (H.I.) .- Asymptotic Dirichlet Problems for Harmonic Functions on Riemannian Manifolds,

Trans. Amer. Math. Soc. 281 (1984), pp. 691-716. 